\title{
Promoting imaging appropriateness in pediatric radiology
}

\section{Cory M. Pfeifer ${ }^{1}$ [D}

Received: 1 October 2019 / Revised: 9 October 2019 / Accepted: 16 October 2019 / Published online: 13 February 2020

(C) Springer-Verlag GmbH Germany, part of Springer Nature 2019

Appropriate utilization of radiology resources has become an important topic in medicine. Not only does inappropriate ordering of radiology exams increase health care costs, but it potentially exposes people to unnecessary radiation. This issue is crucial in pediatric radiology and has formed the core of the Image Gently initiative [1-3]. The concept of imaging appropriateness is so entrenched in health care policy that clinical decision support will soon be mandatory for advanced imaging studies under the Protecting Access to Medicare Act of 2014 [4].

Imaging utilization among pediatric patients differs on an institution-to-institution basis. Lodwick et al. [5] found significant variation in CT utilization across 30 hospitals, and Menoch et al. [6] showed that CT was more likely to be ordered for suspected appendicitis at a private practice tertiarycare emergency department compared to an academic children's hospital. I have observed at my institution an increased use of imaging as a first test without supporting clinical indicators of procedure necessity. This has coincided with increases in imaging requests from physician trainees and physician extenders, with the number of nurse practitioners and physician assistants working in the hospital on the rise.

Presented in this issue is a quality initiative undertaken at my facility designed to bridge the knowledge gap between radiologists and ordering providers by offering a lecture series in partnership with our large pediatric residency program [7]. Didactic lectures that integrate the American College of Radiology (ACR) appropriateness criteria into educational rounds of internal medicine residents were well received during my residency training [8], so the move to pediatrics was a natural transition. In talking with the pediatric chief residents during our needs assessment, it became clear that pediatrics

Cory M. Pfeifer

Cory.Pfeifer@utsouthwestern.edu

1 Department of Radiology, University of Texas Southwestern Medical Center, 5323 Harry Hines Blvd., Dallas, TX 75390, USA residents want to hear more from radiologists regarding cost, radiation exposure, and appropriateness mixed with some basic imaging findings that they might be expected to recognize during their clinical duties. They asked that radiologists take the lead in presenting at their pediatrics lunch-time educational rounds for several sessions.

A particularly valuable part of didactics regarding imaging appropriateness involved showing the ACR appropriateness criteria tables for every indication discussed and, perhaps more important, continuously emphasizing where these data tables can be found for future reference. As a bit of a twist, we included a few case scenarios in which the ordered tests appeared to be suboptimal for the clinical question indicated, and requested suggestions for improvement. Quizzes given to the residents before and after the lecture series showed improved understanding of appropriateness.

Soon, groups of mid-level providers in the hospital asked for similar sets of radiologist-driven lectures to be deployed. Radiologists have now been integrated into the daily rounds of the neonatal intensive care unit as well as other pediatric specialty groups in the hospital, with each session offering elements regarding appropriateness and limitations of imaging on a modality-by-modality basis. By 2019, our medical school had launched similar online radiology appropriateness modules for graduating students, with the pediatric radiology section leading the way. The needs assessment for medical students showed that this population prefers software-based education with test questions that use language reflected in the ACR appropriateness criteria. Similar online modules for firstyear pediatrics residents are currently in development. Learners at all levels have endorsed the value of this type of radiologist-driven education.

What has become apparent and will likely emerge as a new frontier in ensuring appropriate utilization is that access to appropriate imaging may be a limiting factor. For instance, the area of least improved comprehension after the resident lecture series was the persistent belief that head CT should be used as a best first imaging test for pediatric seizures in the absence of trauma. In looking at the utilization of head CT for 
non-trauma-associated seizures in our pediatric emergency departments in 1 month, I found that CT of the head had been ordered on $13 \%$ of children presenting with non-traumaassociated seizures, with that percentage rising to $25 \%$ for those with commercial insurance. While CT of the head has been suggested for partial seizures "when MRI is unavailable or contraindicated" in the comments section of the ACR appropriateness criteria table, CT of the head is notably lesser in its appropriateness compared to MRI. If emergent MRI availability becomes the norm for pediatric emergency departments moving forward, it will be interesting to see whether fewer children undergo CT emergently, particularly if rapid MR techniques [9] continue to improve with respect to diagnostic specificity.

Some caveats to undertaking a program such as the one described here are notable. The presentation style of the ACR appropriateness criteria is changing, with the new style moving away from using a number designation (1-9) for appropriateness. The new versions simply state that each procedure is "usually appropriate," "may be appropriate" or is "usually not appropriate." Also, the comments section of the table is being phased out, meaning that such comments as "if MRI is unavailable or contraindicated" with respect to head CT for the child with partial seizures will be an element of the past. While the ACR appropriateness criteria include relative radiation indicators for each procedure, possible risks of general anesthesia associated with MRI are not addressed.

In conclusion, ordering providers value radiologist-driven initiatives to improve understanding of appropriate utilization of radiology resources. Pediatric radiologists who partner with pediatricians to deliver this desirable content take important steps toward promoting cost savings, limiting radiation to children, and optimizing patient experiences.

\section{Compliance with ethical standards}

Conflicts of interest None

\section{References}

1. Frush DP, Lungren MP (2017) The Image Gently Think-A-Head Campaign: keep calm and image gently. J Am Coll Radiol 14:301302

2. Frush DP, Strauss KJ (2017) Image Gently: getting it right. J Am Coll Radiol 14:575-576

3. Hill KD, Frush DP (2018) Image Gently Have-A-Heart campaign. J Am Coll Radiol 15:372-373

4. Hentel KD, Menard A, Mongan J et al (2019) What physicians and health organizations should know about mandated imaging appropriate use criteria. Ann Intern Med 170:880-885

5. Lodwick DL, Cooper JN, Kelleher KJ et al (2015) Variation in utilization of computed tomography imaging at tertiary pediatric hospitals. Pediatrics 136:e1212-e1219

6. Menoch M, Simon HK, Hirsh D et al (2017) Imaging for suspected appendicitis: variation between academic and private practice models. Pediatr Emerg Care 33:147-151

7. Pfeifer CM, Castillo SM (2020) Pediatric radiologist-driven didactics for a pediatrics residency program: a quality initiative. Pediatr Radio. https://doi.org/10.1007/s00247-019-04559-2

8. Pfeifer CM (2019) The value of radiology resident participation in internal medicine morbidity and mortality conferences at a small center of graduate medical education. Curr Probl Diagn Radiol 48: 305-307

9. Pfeifer CM (2018) Rapid-sequence MRI of the brain: a distinct imaging study. AJNR Am J Neuroradiol 39:E93-E94

Publisher's note Springer Nature remains neutral with regard to jurisdictional claims in published maps and institutional affiliations. 